\title{
On exponential cosmological type solutions in the model with Gauss-Bonnet term and variation of gravitational constant
}

\author{
V. D. Ivashchuk ${ }^{1,2, a}$, A. A. Kobtsev ${ }^{2, b}$ \\ ${ }^{1}$ Center for Gravitation and Fundamental Metrology, VNIIMS, 46 Ozyornaya ul., Moscow 119361, Russia \\ 2 Institute of Gravitation and Cosmology, Peoples' Friendship University of Russia, 6 Miklukho-Maklaya ul., Moscow 117198, Russia
}

Received: 4 March 2015 / Accepted: 3 April 2015 / Published online: 30 April 2015

(C) The Author(s) 2015. This article is published with open access at Springerlink.com

\begin{abstract}
A $D$-dimensional gravitational model with Gauss-Bonnet term is considered. When an ansatz with diagonal cosmological type metrics is adopted, we find solutions with an exponential dependence of the scale factors (with respect to a "synchronous-like" variable) which describe an exponential expansion of "our" 3-dimensional factor space and obey the observational constraints on the temporal variation of effective gravitational constant $G$. Among them there are two exact solutions in dimensions $D=22,28$ with constant $G$ and also an infinite series of solutions in dimensions $D \geq 2690$ with the variation of $G$ obeying the observational data.
\end{abstract}

\section{Introduction}

Here we deal with $D$-dimensional gravitational model with the Gauss-Bonnet term. The action reads

$S=\int_{M} \mathrm{~d}^{D} z \sqrt{|g|}\left\{\alpha_{1} R[g]+\alpha_{2} \mathcal{L}_{2}[g]\right\}$,

where $g=g_{M N} \mathrm{~d} z^{M} \otimes \mathrm{d} z^{N}$ is the metric defined on the manifold $M, \operatorname{dim} M=D,|g|=\left|\operatorname{det}\left(g_{M N}\right)\right|$, and

$\mathcal{L}_{2}=R_{M N P Q} R^{M N P Q}-4 R_{M N} R^{M N}+R^{2}$

is the standard Gauss-Bonnet term. Here $\alpha_{1}$ and $\alpha_{2}$ are nonzero constants.

Earlier the appearance of the Gauss-Bonnet term was motivated by string theory [1-5]. At present, the (so-called) Einstein-Gauss-Bonnet (EGB) gravitational model and its modifications are intensively used in cosmology; see $[6,7]$ (for $D=4$ ) [8-15,17], and references therein, e.g. for the explanation of the accelerating expansion of the Universe following from supernovae (type Ia) observational data [18-20].

\footnotetext{
a e-mail: ivashchuk@mail.ru

b e-mail: speedyblackbird@gmail.com
}

Certain exact solutions in multi-dimensional EGB cosmology were obtained in [8-17] and some other papers.

Here we are dealing with the cosmological type solutions with diagonal metrics (of Bianchi-I-like type) governed by $n$ scale factors depending upon one variable, where $n>$ 3. Moreover, we restrict ourselves to the solutions with an exponential dependence of scale factors (with respect to the "synchronous-like" variable $\tau$ )

$a_{i}(\tau) \sim \exp \left(h^{i} \tau\right)$,

$i=1, \ldots, n ; D=n+1$, with the aim to find solutions describing an exponential isotropic expansion of 3dimensional flat factor space, i.e. with

$h^{1}=h^{2}=h^{3}=H>0$,

and a small enough variation of the effective gravitational constant $G$, which is proportional to the inverse volume scale factor of the internal space, i.e.

$G \sim \prod_{i=4}^{n}\left[a_{i}(\tau)\right]^{-1} \sim \exp (-\operatorname{Int} \tau)$,

where here and in what follows we denote

Int $=\sum_{i=4}^{n} h^{i}$;

see [21-25] and references therein. We call $h^{i}=\dot{a}_{i} / a_{i}$ the "Hubble-like" parameter corresponding to the $i$ th subspace.

In the cosmological model under consideration with anisotropic "internal space", we get for the dimensionless parameter of the temporal variation of $G$ the following relation from (1.5) and (1.6):

$$
\frac{\dot{G}}{G H}=-\mathrm{Int} / H
$$

where $H$ is the Hubble parameter. 
As for the experimental data, the variation of the gravitational constant is allowed at the level of $10^{-13}$ per year and less. We use the following constraint on the magnitude of the dimensionless variation of the gravitational constant:

$-0.65 \times 10^{-3}<\frac{\dot{G}}{G H}<1.12 \times 10^{-3}$,

which comes from the most stringent limitation on $G$-dot obtained by the set of ephemerides [26]

$\dot{G} / G=(0.16 \pm 0.6) \times 10^{-13}$ year $^{-1}$

allowed at $95 \%$ confidence (2- $\sigma$ ) level and the present value of the Hubble parameter [27] (which characterizes the rate of expansion of the observable Universe),

$$
\begin{aligned}
H_{0} & =(67.80 \pm 1.54) \mathrm{km} / \mathrm{s} \mathrm{Mpc}^{-1} \\
& =(6.929 \pm 0.157) \times 10^{-11} \text { year }^{-1},
\end{aligned}
$$

with $95 \%$ confidence level. It should be noted that the original result for $H_{0}$ in [27] (for the Planck best-fit cosmology including an external data set) was presented at $68 \%$ confidence $(1-\sigma)$ level. In the restriction (1.8) we use the lower allowed value for $H_{0}$ in (1.10) in order to obtain the confidence level of more than $95 \%$.

Thus, we are seeking here the cosmological solutions which obey (1.3)-(1.8), listed above.

The paper is organized as follows. In Sect. 2 the equations of motion for the $D$-dimensional EGB model are considered. For diagonal cosmological type metrics the equations of motion are equivalent to a set of Lagrange equations corresponding to a certain "effective" Lagrangian $[29,30]$ (see also $[9,28])$. In Sect. 3 some cosmological solutions with an exponential behavior of the scale factors satisfying the restriction (1.8) are obtained for two isotropic factor spaces and a positive value of $\alpha=\alpha_{2} / \alpha_{1}$.

\section{The cosmological type model and its effective Lagrangian}

\subsection{The set-up}

Here we consider the manifold

$M=\mathbb{R}_{*} \times M_{1} \times \cdots \times M_{n}$,

with the metric

$g=w \mathrm{e}^{2 \gamma(u)} \mathrm{d} u \otimes \mathrm{d} u+\sum_{i=1}^{n} \mathrm{e}^{2 \beta^{i}(u)} \varepsilon_{i} \mathrm{~d} y^{i} \otimes \mathrm{d} y^{i}$, where $w= \pm 1, \varepsilon_{i}= \pm 1, i=1, \ldots, n$, and $M_{1}, \ldots, M_{n}$ are 1-dimensional manifolds (either $\mathbb{R}$ or $S^{1}$ ). Here and in what follows $\mathbb{R}_{*}=\left(u_{-}, u_{+}\right)$is an open subset in $\mathbb{R}$. The functions $\gamma(u)$ and $\beta^{i}(u), i=1, \ldots, n$, are smooth on $\mathbb{R}_{*}=\left(u_{-}, u_{+}\right)$.

For $w=-1, \varepsilon_{1}=\cdots=\varepsilon_{n}=1$ the metric (2.2) is a cosmological one, while for $w=1, \varepsilon_{1}=-1, \varepsilon_{2}=\cdots=$ $\varepsilon_{n}=1$ it describes certain static configurations.

For physical applications we put $M_{1}=M_{2}=M_{3}=\mathbb{R}$, while $M_{4}, \ldots, M_{n}$ will be considered to be compact ones (i.e. coinciding with $S^{1}$ ).

The integrand in (1.1), when the metric (2.2) is substituted, reads as follows:

$\sqrt{|g|}\left\{\alpha_{1} R[g]+\alpha_{2} \mathcal{L}_{2}[g]\right\}=L+\frac{\mathrm{d} f}{\mathrm{~d} u}$,

where

$L=\alpha_{1} L_{1}+\alpha_{2} L_{2}$,

$L_{1}=(-w) \mathrm{e}^{-\gamma+\gamma_{0}} G_{i j} \dot{\beta}^{i} \dot{\beta}^{j}$,

$L_{2}=-\frac{1}{3} \mathrm{e}^{-3 \gamma+\gamma_{0}} G_{i j k l} \dot{\beta}^{i} \dot{\beta}^{j} \dot{\beta}^{k} \dot{\beta}^{l}$,

$\gamma_{0}=\sum_{i=1}^{n} \beta^{i}$,

and

$G_{i j}=\delta_{i j}-1$,

$G_{i j k l}=\left(\delta_{i j}-1\right)\left(\delta_{i k}-1\right)\left(\delta_{i l}-1\right)\left(\delta_{j k}-1\right)\left(\delta_{j l}-1\right)\left(\delta_{k l}-1\right)$

are, respectively, the components of the 2-metrics on $\mathbb{R}^{n}$ $[29,30]$. The first one is the well-known "minisupermetric" 2-metric of pseudo-Euclidean signature: $\left\langle v_{1}, v_{2}\right\rangle=$ $G_{i j} v_{1}^{i} v_{2}^{j}$, and the second one is the Finslerian 4-metric: $\left\langle v_{1}, v_{2}, v_{3}, v_{4}\right\rangle=G_{i j k l} v_{1}^{i} v_{2}^{j} v_{3}^{k} v_{4}^{l}, v_{s}=\left(v_{s}^{i}\right) \in \mathbb{R}^{n}$, where $\langle.,$.$\rangle and \langle., ., .,$.$\rangle are, respectively, 2- and 4-linear symmet-$ ric forms on $\mathbb{R}^{n}$. Here we denote $\dot{A}=\mathrm{d} A / \mathrm{d} u$ etc. The function $f(u)$ in (2.3) is irrelevant for our considerations (see $[29,30])$.

The derivation of (2.4)-(2.6) is based on the following identities [29,30]:

$$
\begin{aligned}
& G_{i j} v^{i} v^{j}=\sum_{i=1}^{n}\left(v^{i}\right)^{2}-\left(\sum_{i=1}^{n} v^{i}\right)^{2}, \\
& G_{i j k l} v^{i} v^{j} v^{k} v^{l}=\left(\sum_{i=1}^{n} v^{i}\right)^{4}-6\left(\sum_{i=1}^{n} v^{i}\right)^{2} \sum_{j=1}^{n}\left(v^{j}\right)^{2} \\
& +3\left(\sum_{i=1}^{n}\left(v^{i}\right)^{2}\right)^{2}+8\left(\sum_{i=1}^{n} v^{i}\right) \sum_{j=1}^{n}\left(v^{j}\right)^{3}-6 \sum_{i=1}^{n}\left(v^{i}\right)^{4} .
\end{aligned}
$$


It follows immediately from the definitions (2.8) and (2.9) that

$$
\begin{aligned}
& G_{i j} v^{i} v^{j}=-2 \sum_{i<j} v^{i} v^{j}, \\
& G_{i j k l} v^{i} v^{j} v^{k} v^{l}=24 \sum_{i<j<k<l} v^{i} v^{j} v^{k} v^{l} .
\end{aligned}
$$

\subsection{The equations of motion}

The equations of motion corresponding to the action (1.1) have the following form:

$\mathcal{E}_{M N}=\alpha_{1} \mathcal{E}_{M N}^{(1)}+\alpha_{2} \mathcal{E}_{M N}^{(2)}=0$,

where

$$
\begin{aligned}
\mathcal{E}_{M N}^{(1)}= & R_{M N}-\frac{1}{2} \operatorname{Rg}_{M N}, \\
\mathcal{E}_{M N}^{(2)}= & 2\left(R_{M P Q S} R_{N}{ }^{P Q S}-2 R_{M P} R_{N}{ }^{P}\right. \\
& \left.-2 R_{M P N Q} R^{P Q}+R R_{M N}\right)-\frac{1}{2} \mathcal{L}_{2} g_{M N} .
\end{aligned}
$$

It was shown in [30] that the field equations (2.14) for the metric (2.2) are equivalent to the Lagrange equations corresponding to the Lagrangian $L$ from (2.4).

Thus, Eq. (2.14) read as follows:

$$
\begin{aligned}
& w \alpha_{1} G_{i j} \dot{\beta}^{i} \dot{\beta}^{j}+\alpha_{2} \mathrm{e}^{-2 \gamma} G_{i j k l} \dot{\beta}^{i} \dot{\beta}^{j} \dot{\beta}^{k} \dot{\beta}^{l}=0, \\
& \frac{\mathrm{d}}{\mathrm{d} u}\left[-2 w \alpha_{1} G_{i j} \mathrm{e}^{-\gamma+\gamma_{0}} \dot{\beta}^{j}-\frac{4}{3} \alpha_{2} \mathrm{e}^{-3 \gamma+\gamma_{0}} G_{i j k l} \dot{\beta}^{j} \dot{\beta}^{k} \dot{\beta}^{l}\right]-L \\
& \quad=0, \\
& i=1, \ldots, n \text {. Due to (2.17) } L=-w \frac{2}{3} \mathrm{e}^{-\gamma+\gamma_{0}} \alpha_{1} G_{i j} \dot{\beta}^{i} \dot{\beta}^{j} \text {. }
\end{aligned}
$$

\subsection{Reduction to an autonomous system of first-order} differential equations

Now we put $\gamma=0$ and denote $u=\tau$, where $\tau$ is a "synchronous-like" variable. By introducing "Hubble-like" variables $h^{i}=\dot{\beta}^{i}$, Eqs. (2.17) and (2.18) may be rewritten as follows:

$$
\begin{aligned}
& w \alpha_{1} G_{i j} h^{i} h^{j}+\alpha_{2} G_{i j k l} h^{i} h^{j} h^{k} h^{l}=0, \\
& {\left[-2 w \alpha_{1} G_{i j} h^{j}-\frac{4}{3} \alpha_{2} G_{i j k l} h^{j} h^{k} h^{l}\right] \sum_{i=1}^{n} h^{i}} \\
& +\frac{\mathrm{d}}{\mathrm{d} \tau}\left[-2 w \alpha_{1} G_{i j} h^{j}-\frac{4}{3} \alpha_{2} G_{i j k l} h^{j} h^{k} h^{l}\right]-L=0,
\end{aligned}
$$

$i=1, \ldots, n$, where

$L=-w \alpha_{1} G_{i j} h^{i} h^{j}-\frac{1}{3} \alpha_{2} G_{i j k l} h^{i} h^{j} h^{k} h^{l}$.

Due to (2.19) $L=-\frac{2}{3} w \alpha_{1} G_{i j} h^{i} h^{j}$.
Thus, we are led to the autonomous system of the firstorder differential equations on $h^{1}(\tau), \ldots, h^{n}(\tau)[30,30]$.

Here and in what follows we use Eqs. (2.10), (2.11), and the following formulas:

$$
\begin{aligned}
G_{i j} v^{j}= & v^{i}-S_{1}, \\
G_{i j k l} v^{j} v^{k} v^{l}= & S_{1}^{3}+2 S_{3}-3 S_{1} S_{2}+3\left(S_{2}-S_{1}^{2}\right) v^{i} \\
& +6 S_{1}\left(v^{i}\right)^{2}-6\left(v^{i}\right)^{3},
\end{aligned}
$$

$i=1, \ldots, n$, where $S_{k}=S_{k}(v)=\sum_{i=1}^{n}\left(v^{i}\right)^{k}$.

\subsection{Solutions with constant $h^{i}$}

In this paper we deal with the following solutions to (2.19) and (2.20):

$h^{i}(\tau)=v^{i}$,

with constant $v^{i}$, which correspond to the solutions

$\beta^{i}=v^{i} \tau+\beta_{0}^{i}$,

where $\beta_{0}^{i}$ are constants, $i=1, \ldots, n$.

In this case we obtain the metric (2.2) with the exponential dependence of the scale factors

$g=w \mathrm{~d} \tau \otimes \mathrm{d} \tau+\sum_{i=1}^{n} \varepsilon_{i} B_{i}^{2} \mathrm{e}^{2 v^{i} \tau} \mathrm{d} y^{i} \otimes \mathrm{d} y^{i}$,

where $w= \pm 1, \varepsilon_{i}= \pm 1$, and $B_{i}>0$ are arbitrary constants.

For the fixed point $v=\left(v^{i}\right)$ we have the set of polynomial equations

$G_{i j} v^{i} v^{j}-\alpha G_{i j k l} v^{i} v^{j} v^{k} v^{l}=0$,

$\left[2 G_{i j} v^{j}-\frac{4}{3} \alpha G_{i j k l} v^{j} v^{k} v^{l}\right] \sum_{i=1}^{n} v^{i}-\frac{2}{3} G_{i j} v^{i} v^{j}=0$,

$i=1, \ldots, n$, where $\alpha=\alpha_{2}(-w) / \alpha_{1}$. For $n>3$ this is a set of fourth-order polynomial equations.

The trivial solution $v=\left(v^{i}\right)=(0, \ldots, 0)$ corresponds to a flat metric $g$.

For any non-trivial solution $v$ we have $\sum_{i=1}^{n} v^{i} \neq 0$ [otherwise one gets from (2.28) $G_{i j} v^{i} v^{j}=\sum_{i=1}^{n}\left(v^{i}\right)^{2}-$ $\left(\sum_{i=1}^{n} v^{i}\right)^{2}=0$ and hence $\left.v=(0, \ldots, 0)\right]$.

The set of equations (2.27) and (2.28) has an isotropic solution $v^{1}=\cdots=v^{n}=H$, where

$n(n-1) H^{2}+\alpha n(n-1)(n-2)(n-3) H^{4}=0$.

For $n=1: H$ is arbitrary and for $n=2,3: H=0$. 
When $n>3$, the non-zero solution to Eq. (2.29) exists only if $\alpha<0$ and in this case [29,30]

$H= \pm \frac{1}{\sqrt{|\alpha|(n-2)(n-3)}}$.

In the cosmological case $(w=-1)$ this solution occurs when $\alpha_{2} / \alpha_{1}=\alpha<0$.

The isotropic solution for $n>3$ gives rise to a very large value of $\dot{G} / G=(n-3) H$, which is forbidden by observational restrictions.

It was shown in $[29,30]$ that there are no more than three different numbers among $v^{1}, \ldots, v^{n}$.

\section{Examples of cosmological solutions obeying the restriction on the variation of $G$}

In this section we consider some solutions to the set of Eqs. (2.27) and (2.28) of the following form: $v=$ $(H, \ldots, H, h, \ldots, h)$, where $H$ the "Hubble-like" parameter corresponding to $m$-dimensional isotropic subspace with $m>3$ and $h$ is the "Hubble-like" parameter corresponding to the $l$-dimensional isotropic subspace, $l>2$.

These solutions should satisfy the following conditions:

1. mandatory:

(a) $H$ and $h$ are real numbers,

(b) $H>0, h<0$;

2. desirable:

(a) Int $=(m-3) H+l h<0$;

(b) $-0.65 \times 10^{-3}<\frac{\dot{G}}{G H}=-\left((m-3)+\frac{l h}{H}\right)<1.12 \times$ $10^{-3}$.

The first inequality, $H>0$, in the mandatory condition is necessary for a description of accelerated expansion of 3-dimensional subspace, which may describe our Universe, while the second inequality, $h<0$, excludes an enormous (of the order of the Hubble parameter) variation $\dot{G} / G$ for $h \geq 0$ and $m>3$.

The first desirable condition means that the volume scale factor of the internal space $V(\tau)=B \exp (((m-3) H+l h) \tau)$, where $B>0$ is constant, decreases over time. This condition is a sort of weak extension of a possible restriction for $m=3$ coming from the unobservability of the "internal space" for all $\tau>\tau_{0}$. It is also desirable since the negative value of the parameter Int is more probable due to the more probable positive value of $\dot{G} / G=-I n t$; see (1.9).

\footnotetext{
1 At the moment we were unable to find solutions with three different real "Hubble-like" parameters.
}

The second desirable condition may also be rewritten by using the parameter $\operatorname{Var}=\left|\frac{\dot{G}}{G H}\right|=\left|(m-3)+\frac{l h}{H}\right|$ :

$\operatorname{Var}<1.12 \times 10^{-3}$, for Int $\leq 0$;

Var $<0.65 \times 10^{-3}$, for Int $\geq 0$.

Here we consider the simplest case, when the internal spaces (apart from the expansion factors) are flat. The consideration of curved internal spaces will drastically change the equations of motion and may break the existence of solutions with an exponential dependence of the scale factors. Anyway, the inclusion into our consideration of curved internal spaces may be worthwhile, but it needs a special treatment, which may be given in a separate work.

\subsection{The dependence of "Hubble-like" parameters on $m$ and $l$}

The total dimension of the considered space is $D=n+1=$ $(m+l)+1$, where we have $m$ dimensions expanding with the Hubble parameter $H>0$ and $l$ dimensions contracting with the "Hubble-like" parameter $h<0$.

According to this, we rewrite the set of polynomial equations (2.27), (2.28), using Eqs. (2.22) and (2.23), as follows:

$$
\begin{aligned}
& H^{2}\left(m-m^{2}\right)+h^{2}\left(l-l^{2}\right)-2 m l H h \\
& \quad-\alpha\left(H^{4} m(m-1)(m-2)(m-3)+h^{4} l(l-1)\right. \\
& \quad \times(l-2)(l-3) \\
& +4 H^{3} h m(m-1)(m-2) l+4 h^{3} H l(l-1)(l-2) m \\
& \left.+6 H^{2} h^{2} m(m-1) l(l-1)\right)=0,
\end{aligned}
$$

$$
\begin{aligned}
& m(1-m) H^{2}-(1 / 2) l h^{2}(1+2 l)+2 l H h((3 / 4)-m) \\
& \quad-\alpha\left(H^{4} m(m-1)(m-2)(m-3)+H^{3} h l(m-1)\right. \\
& \quad \times(m-2)(4 m-3) \\
& \quad+3 H^{2} h^{2} l(m-1)(2 l m-2 l-m) \\
& \left.\quad+H h^{3} l(l-1)(4 l m-3 l-2 m)+h^{4} l^{2}(l-1)(l-2)\right)=0,
\end{aligned}
$$

$$
\begin{aligned}
& l(1-l) h^{2}-(1 / 2) m H^{2}(1+2 m)+2 m H h((3 / 4)-l) \\
& \quad-\alpha\left(h^{4} l(l-1)(l-2)(l-3)+h^{3} H m(l-1)(l-2)(4 l-3)\right. \\
& \quad+3 h^{2} H^{2} m(l-1)(2 l m-2 m-l) \\
& +h H^{3} m(m-1)(4 l m-3 m-2 l)+H^{4} m^{2}(m-1) \\
& \quad \times(m-2))=0 .
\end{aligned}
$$

Here we put for simplicity $\alpha= \pm 1$ but keep in mind that general $\alpha$-dependent solution has the following form:

$H(\alpha)=H|\alpha|^{-1 / 2}, \quad h(\alpha)=h|\alpha|^{-1 / 2}$.

Due to these relations the parameter $\dot{G} /(G H)$ does not depend upon $|\alpha|$ and hence our simplification is a reasonable 
one. For any solution $(H, h)$ with $\alpha= \pm 1$ we can find a proper $\alpha$, which will be in agreement with the present value of the Hubble parameter $H_{0}$ [see (1.10)]

$H|\alpha|^{-1 / 2}=H_{0}$

Our numerical analysis (based on Maplesoft Maple) shows that (generically) there are 11 solutions of these equations (for $m>3$ and $l \geq 3$ ).

(I) The first to mention is, obviously, the zero solution $H_{1}=$ $h_{1}=0$.

(II) Two other solutions are isotropic ones:

1. if $\alpha=1$, then $H=h= \pm \sqrt{\frac{1}{\left(l^{2}+2 l m+m^{2}-5 l-5 m+6\right)}}$. $i$. We are led to pure imaginary isotropic solutions obeying (2.29). For example, when $m=9$ and $l=6$ we obtain

(a) $H_{2}=h_{2}=\frac{1}{2} \sqrt{\frac{1}{39}} \cdot i$
(b) $H_{3}=h_{3}=-\frac{1}{2} \sqrt{\frac{1}{39}} \cdot i$

2. if $\alpha=-1$, then $H=h= \pm \sqrt{\frac{1}{\left(l^{2}+2 l m+m^{2}-5 l-5 m+6\right)}}$. We are led to isotropic solutions (2.30). When $m=9$ and $l=6$ the solutions are:
(a) $H_{2}=h_{2}=\frac{1}{2} \sqrt{\frac{1}{39}}$;
(b) $H_{3}=h_{3}=-\frac{1}{2} \sqrt{\frac{1}{39}}$.

(III) For $\alpha= \pm 1$ the remaining eight solutions are roots of the following two equations which are given by Maple:

$$
\begin{aligned}
P(H ; m, l)= & 64(m-2)(m-1)^{2}(m-2+l)\left(l^{2} m+l m^{2}\right. \\
& \left.-2 l^{2}+2 l m-2 m^{2}\right)(-3+l+m)^{2} \cdot H^{8} \\
& \mp 128(m-1)^{2}(-3+l+m)\left(l^{2} m(l+m)^{2}\right. \\
& -2 l(l+m)^{3}+(l+m)\left(8 l^{2}+l m+2 m^{2}\right) \\
& \left.-10 l^{2}+4 l m-4 m^{2}\right) \cdot H^{6} \\
& +16(m-1)\left(5 l^{5} m+10 l^{4} m^{2}+5 l^{3} m^{3}\right. \\
& -6 l^{5}-38 l^{4} m-49 l^{3} m^{2}-17 l^{2} m^{3}+32 l^{4} \\
& +111 l^{3} m+75 l^{2} m^{2}+14 l m^{3}-70 l^{3} \\
& -130 l^{2} m-14 l m^{2} \\
& \left.-8 m^{3}+68 l^{2}+4 l m+8 m^{2}\right) \cdot H^{4} \\
& \mp 16 l(m-1)\left(l^{4}+l^{3} m-3 l^{3}-5 l^{2} m\right. \\
& \left.+5 l^{2}+8 l m-7 l-2 m\right) \cdot H^{2} \\
& +l^{5}-3 l^{3}-2 l^{2}=0, \\
P(h ; l, m)= & 0 .
\end{aligned}
$$

The second equation is obtained from the first one just by swapping the parameters $m$ and $l$ and replacing $H$ by $h$. The solutions to Eqs. (3.8) and (3.9) should be substituted into Eqs. (3.3), (3.4) and (3.5), in order to find the solutions $(H, h)$ under consideration.

The closed-form expression for the solution in general case (for any $m$ and $l$ ) seems to be very bulky. So, we use
Maplesoft Maple to find solutions for certain $m$ and $l$ and test some general features of these solutions: ${ }^{2}$

1. For $\alpha=1$ in the common case we have two pairs of real and complex solutions which differ in signs. (See Footnote 2.) For $m=9$ and $l=6$ we obtain

(a) $H_{4} \approx-0.2597$ and $h_{4} \approx 0.2826$;

(b) $H_{5} \approx 0.2597$ and $h_{5} \approx-0.2826$;

(c) $H_{6} \approx-0.1610$ and $h_{6} \approx 0.4004$;

(d) $H_{7} \approx 0.1610$ and $h_{7} \approx-0.4004$;

(e) $H_{8} \approx-0.0913-0.0464 \cdot i$ and $h_{8} \approx 0.1456-$ $0.0449 \cdot i$

(f) $H_{9} \approx-0.0913+0.0464 \cdot i$ and $h_{9} \approx 0.1456+$ $0.0449 \cdot i$

(g) $H_{10} \approx 0.0913-0.0464 \cdot i$ and $h_{10} \approx-0.1456-$ $0.0449 \cdot i$

(h) $H_{11} \approx 0.0913+0.0464 \cdot i$ and $h_{11} \approx-0.1456+$ $0.0449 \cdot i$

2. for $\alpha=-1$ ( $m>3$ and $l \geq 3$ ) there are no (extra) real solutions. (See Footnote 2.)

In the case of $m=9$ and $l=6$ we obtain

(a) $H_{4} \approx-0.2597 \cdot i$ and $h_{4} \approx 0.2826 \cdot i$;

(b) $H_{5} \approx 0.2597 \cdot i$ and $h_{5} \approx-0.2826 \cdot i$;

(c) $H_{6} \approx 0.1610 \cdot i$ and $h_{6} \approx-0.4004 \cdot i$;

(d) $H_{7} \approx-0.1610 \cdot i$ and $h_{7} \approx 0.4004 \cdot i$;

(e) $H_{8} \approx-0.0464-0.0913 \cdot i$ and $h_{8} \approx-0.0449+$ $0.1456 \cdot i$

(f) $H_{9} \approx-0.0464+0.0913 \cdot i$ and $h_{9} \approx-0.0449-$ $0.1456 \cdot i$;

(g) $H_{10} \approx 0.0464-0.0913 \cdot i$ and $h_{10} \approx 0.0449+$ $0.1456 \cdot i$;

(h) $H_{11} \approx 0.0464+0.0913 \cdot i$ and $h_{11} \approx 0.0449-$ $0.1456 \cdot i$.

It can be seen that none of the solutions for $\alpha=-1$ satisfies our mandatory conditions written in the beginning of this section.

As for $\alpha=1$, the solutions III.1.b and III.1.d are real and $H>0, h<0$. It can be verified that in these cases Int $=(m-3) H+l h<0$.

Now we have to calculate the variation of the gravitational constant. For $m=9$ and $l=6$ we get

$\operatorname{Var}_{5}=\left|\frac{\dot{G}}{G H}\right|_{5}=\left|(m-3)+\frac{l \cdot h_{5}}{H_{5}}\right| \approx 0.535826 ;$

$\operatorname{Var}_{7}=\left|\frac{\dot{G}}{G H}\right|_{7}=\left|(m-3)+\frac{l \cdot h_{7}}{H_{7}}\right| \approx 8.914741$.

\footnotetext{
${ }^{2}$ Here we are led to some common features of the solutions just by
} numerical calculations for a restricted range of numbers $m$ and $l$. 

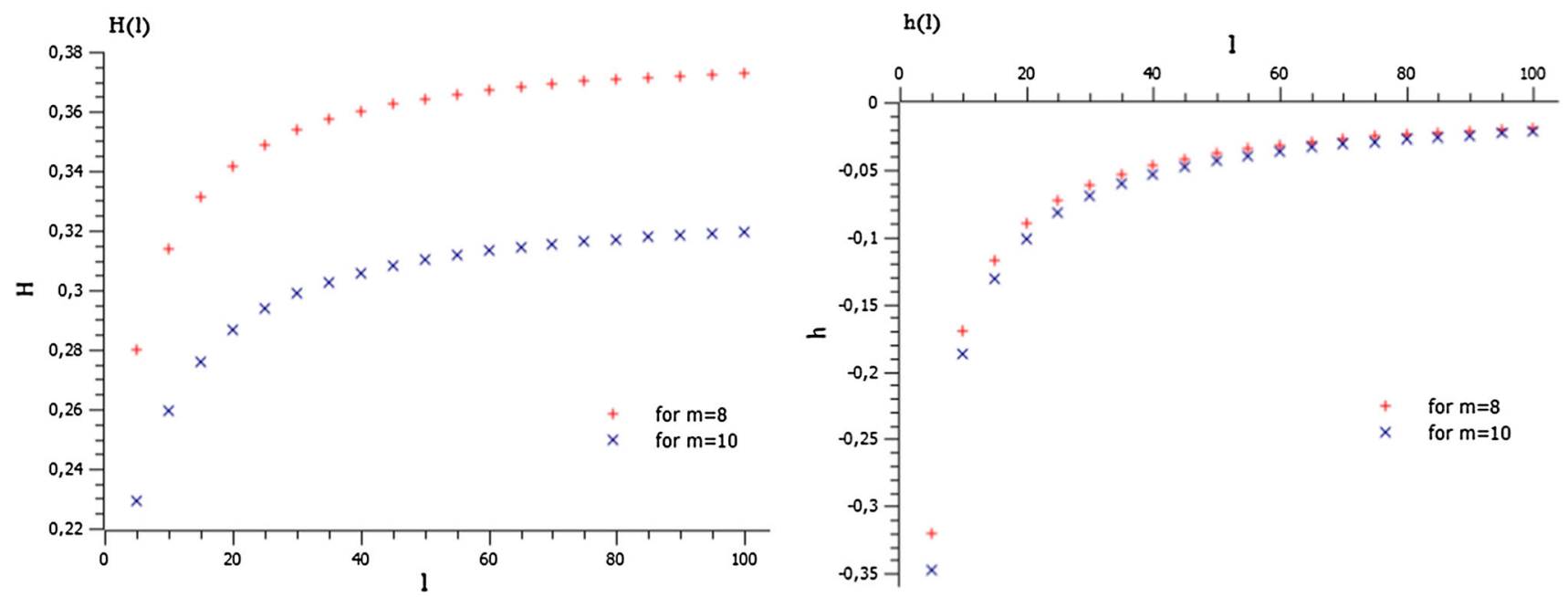

Fig. 1 Behavior of "Hubble-like" parameters $H$ and $h$ for fixed $m=8$ and $m=10$ while $l$ is changing
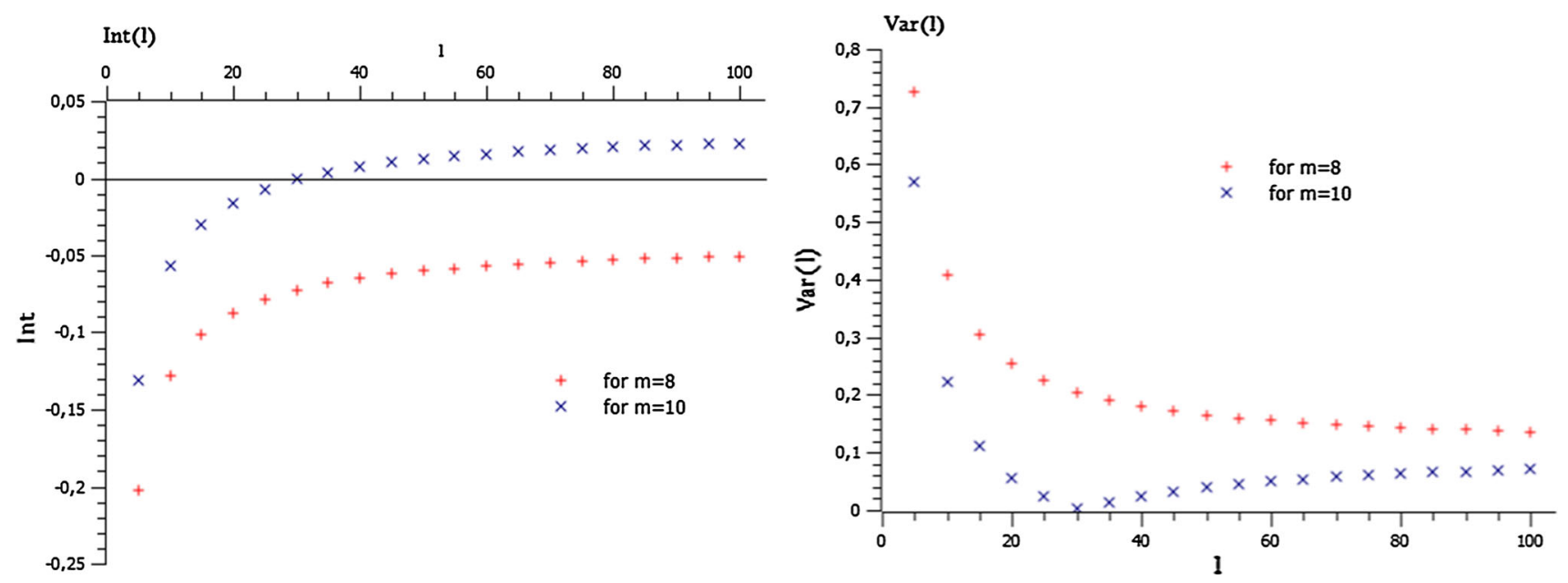

Fig. 2 Behavior of the internal space parameter Int and the variation of $G$ parameter Var for fixed $m=8$ and $m=10$ while $l$ is changing

The first variation is lower: $\operatorname{Var}_{5}<\operatorname{Var}_{7}$, for $m=9$ and $l=6$. This inequality seems to occur for any $m>3$ and $l \geq 3$. At the moment a rigorous proof of this fact is absent, while certain numerical calculations support it. Anyway, here we will focus on the solution $\left(H_{5}, h_{5}\right)$, which we consider as more interesting (for our applications) than $\left(H_{7}, h_{7}\right)$. Further we will write $H$ and $h$ instead of $H_{5}$ and $h_{5}$ in common cases.

We can plot the behavior of the parameters $H, h$, Int, and Var, for example, keeping fixed $m=8$ and $m=10$ and raising $l$ from 5 to 100 by 5 . See Figs. 1 and 2 .

\subsection{The limiting values of $H, h l$, Int, and Var for fixed $m \leq 9$}

When $m \leq 9$ the internal space parameter Int remains negative, which means that the first desirable condition is satisfied for any $l$. The variation of the $G$ parameter is monotonically decreasing with the increase of $l$. Moreover, we get finite limits for $H$ and $h l$ as $l \rightarrow+\infty$. In this subsection we obtain these and other limits (for Int and Var) for fixed $m \leq 9$.

Now let us rewrite (3.8) and (3.9) for $H$ and $h l$ keeping only the terms with higher degrees of $l$ :

$$
\begin{aligned}
& 64(m-1)^{2}(m-2)^{2} l^{5} \cdot H^{8}-128(m-2)(m-1)^{2} l^{5} \cdot H^{6} \\
& +16(m-1)(5 m-6) l^{5} \cdot H^{4}-16(m-1) l^{5} \cdot H^{2}+l^{5}=0, \\
& 64(m-1)(l \cdot h)^{8}-128\left(m^{2}-2 m+2\right)(l \cdot h)^{6} \\
& \quad+\left(80 m^{3}-272 m^{2}+224 m-128\right)(l \cdot h)^{4} \\
& \quad-16 m\left(m^{3}-5 m^{2}+8 m-2\right)(l \cdot h)^{2} \\
& +m^{5}-3 m^{3}-2 m^{2}=0 .
\end{aligned}
$$

Solving these equations we find the limiting values:

$\lim _{l \rightarrow \infty} H=\frac{1}{2} \sqrt{\frac{2 m-2+\sqrt{2 m^{2}-2 m}}{m^{2}-3 m+2}}$, 
Table 1 The limiting values of $H, h l$, Int, and Var parameters as $l \rightarrow \infty$

\begin{tabular}{lllll}
\hline$m$ & $\lim _{l \rightarrow \infty} H$ & $\lim _{l \rightarrow \infty} l \cdot h$ & $\lim _{l \rightarrow \infty} \operatorname{Int}$ & $\lim _{l \rightarrow \infty} \operatorname{Var}$ \\
\hline 3 & 0.9659258265 & -0.7630807575 & -0.7630807575 & 0.7899993318 \\
4 & 0.6738873385 & -1.093021916 & -0.4191345775 & 0.621965355 \\
5 & 0.5462858555 & -1.352249104 & -0.259677393 & 0.475350754 \\
6 & 0.4709825726 & -1.574449592 & -0.161501874 & 0.342904141 \\
7 & 0.4199717390 & -1.772664074 & -0.092777118 & 0.220912766 \\
8 & 0.3825276619 & -1.953613607 & -0.040975297 & 0.107117214 \\
9 & 0.3535533906 & -2.121320344 & 0 & 0 \\
\hline
\end{tabular}

Int $\approx-0.000395288$,

Var $\approx 0.001119293$.

For $l=2682$ :

$H \approx 0.3531586532$,

$h \approx-0.0007902114318$,

Int $\approx-0.000395141$,

Var $\approx 0.001118876$.

We do not present here the exact analytical forms of these solutions in radicals which are bulky ones. For example, the relation for the parameter $H$, when $l=2680$, contains $(17$ times $)$ the radical $\sqrt{32839778319264444823234828568184005}$.

The numerical calculations for fixed $m=9$ gives evidence of the monotonically decreasing behavior of the function $\operatorname{Var}(l)$ for $l \geq 2680^{3}$ as well as the asymptotical relation: $\operatorname{Var}(l) \sim A / l$, as $l \rightarrow+\infty$, where $A>0$. See Fig. 3 .

Thus, for $m=9$ there is an infinite series of admissible cosmological solutions with $l=2680,2681, \ldots$, which satisfy all the conditions imposed. Any such solution describes an accelerated expansion of the 3-dimensional factor space with sufficiently small value of the variation of the effective gravitational constant $G$. This variation may be arbitrarily small for a big enough value of $l$.

The infinite series of solutions for $m=9$ and $l=$ $2680,2681, \ldots$ starts from the (special) total dimension $D=2690$. For $D<2690$ and $m=9$ the solutions do not obey restriction (1.8) on the variation of $G$ and hence are not of interest for our consideration.

3.4 Some solutions for $m>9$ with minimal Var-parameter

When $m>9$ the internal space parameter Int becomes positive. As $l$ can only be a natural number we should look for a value of $l$, which gives the minimal magnitude of the variation of $G$ parameter Var. Below we present the calculated

\footnotetext{
3 A rigorous analytical proof of this fact may be a subject of a separate work.
} 

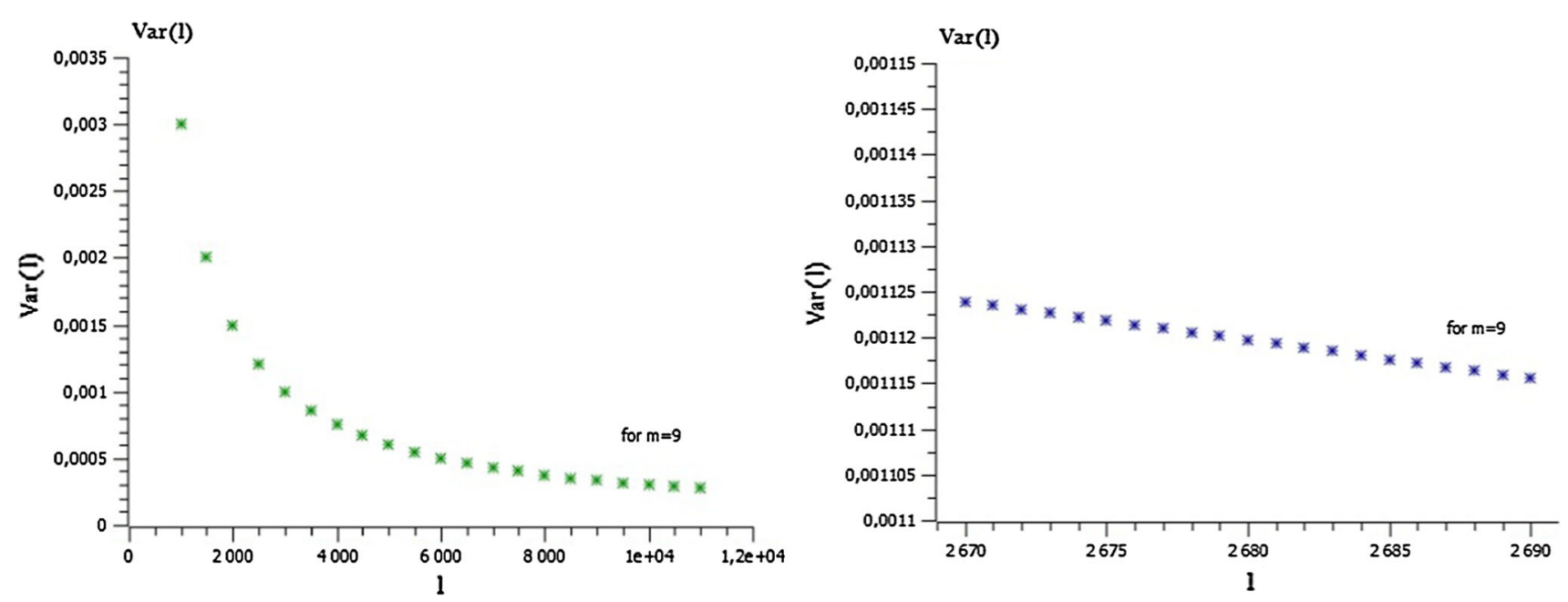

Fig. 3 Behavior of the variation of $G$ parameter Var for fixed $m=9$ while $l$ is changing from 1000 to 11,000 by 500 (left) and from 2670 to 2690 by 1 (right)

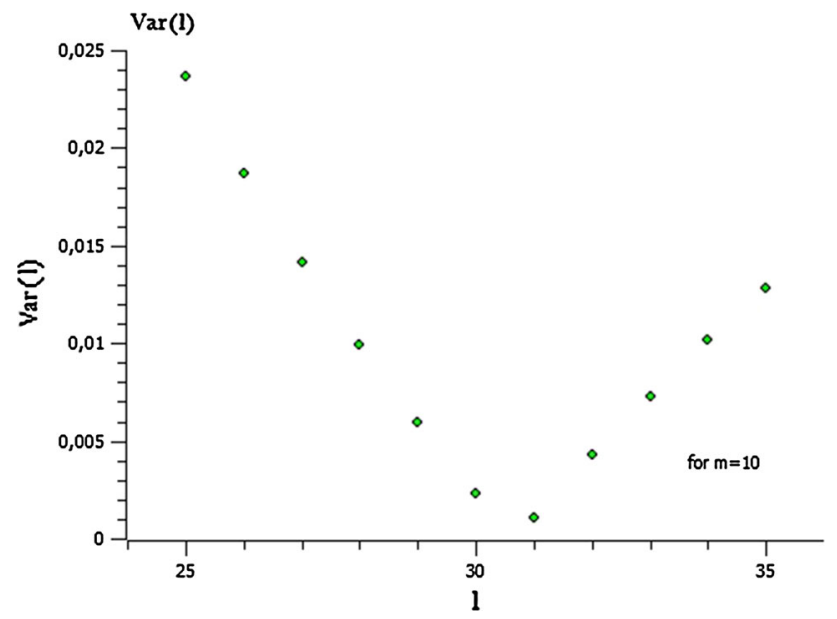

Fig. 4 The variation of the $G$ parameter for $m=10$

values of our parameters $(H, h$, Int $=(m-3) \cdot H+l \cdot h$, and $\left.\operatorname{Var}=\left|(m-3)+\frac{l \cdot h}{H}\right|\right)$ for each of the considered cases:

1. For $m=10$ : the variation of $G$ parameter is minimal for $l=31$, see Fig. 4,

$H \approx 0.2996055415$,

$h \approx-0.06764217686$,

Int $\approx 0.000331307$,

$\operatorname{Var} \approx 0.001105812$.

This case is not of particular interest. The radical forms of the solutions are too bulky, so we approximated them. Nevertheless the variation of the $G$ parameter is out of the allowed domain and the "internal space" parameter is positive.

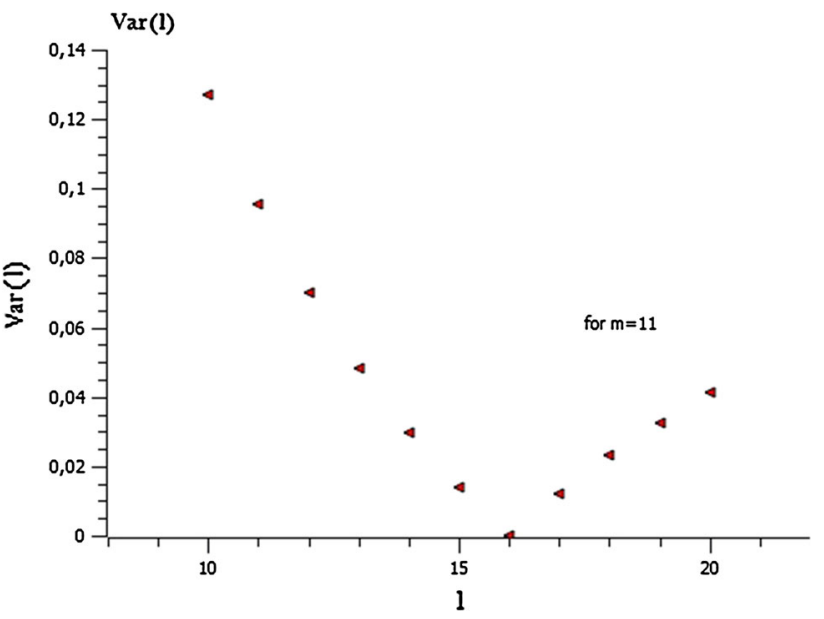

Fig. 5 The variation of the $G$ parameter for $m=11$

2. For $m=11$ : the variation of $G$ and the "internal space" parameters are zero for $l=16$, see Fig. 5,

$H=\sqrt{\frac{1}{15}}$,

$h=-\frac{1}{2} \sqrt{\frac{1}{15}}$,

Int $=0$,

$\operatorname{Var}=0$.

This case is the first one with zero variation of $G$. Also, the exact values of the "Hubble-like" parameters $(H=-2 h)$ in contrast to the previous case have rather simple and compact forms.

3. For $m=12$ : the variation of the $G$ parameter is minimal for $l=11$, see Fig. 6, 


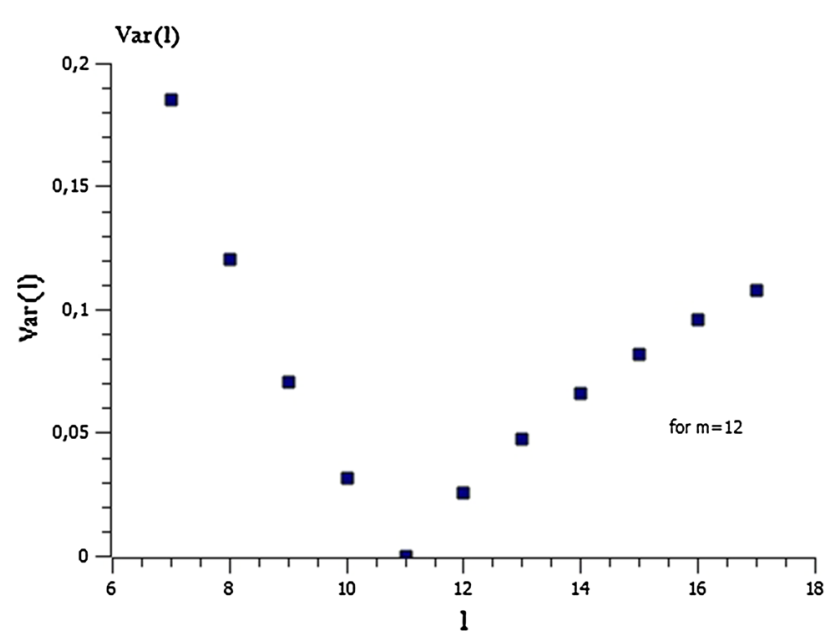

Fig. 6 The variation of the $G$ parameter for $m=12$

$$
\begin{aligned}
H & \approx 0.2264080186, \\
h & \approx-0.1852491999, \\
\text { Int } & \approx-0.000069032, \\
\text { Var } & \approx 0.000304899 .
\end{aligned}
$$

Here all our four conditions are satisfied. The variation of the $G$ parameter is non-zero and the volume of the internal space is decreasing.

4. For $m=13$ : the variation of the $G$ parameter is minimal for $l=9$ and the "internal space" parameter is positive; see Fig. 7,

$$
\begin{aligned}
H & \approx 0.2039802, \\
h & \approx-0.2261006, \\
\text { Int } & \approx 0.0048967, \\
V a r & \approx 0.0240058 .
\end{aligned}
$$

For $l=8$ the variation of the $G$ parameter is slightly higher, but the "internal space" parameter is negative, see Fig. 8.

$$
\begin{aligned}
H & \approx 0.1942063, \\
h & \approx-0.2498379, \\
\text { Int } & \approx-0.0052611, \\
\operatorname{Var} & \approx 0.0263919 .
\end{aligned}
$$

Both cases are excluded by the $G$-dot restrictions.

5. For $m=14$ : the variation of the $G$ parameter is minimal for $l=7$, see Fig. 8 .

$H \approx 0.1822582965$,

$h \approx-0.2863787788$,

Int $\approx 0.000189810$,

$\operatorname{Var} \approx 0.00104143$.

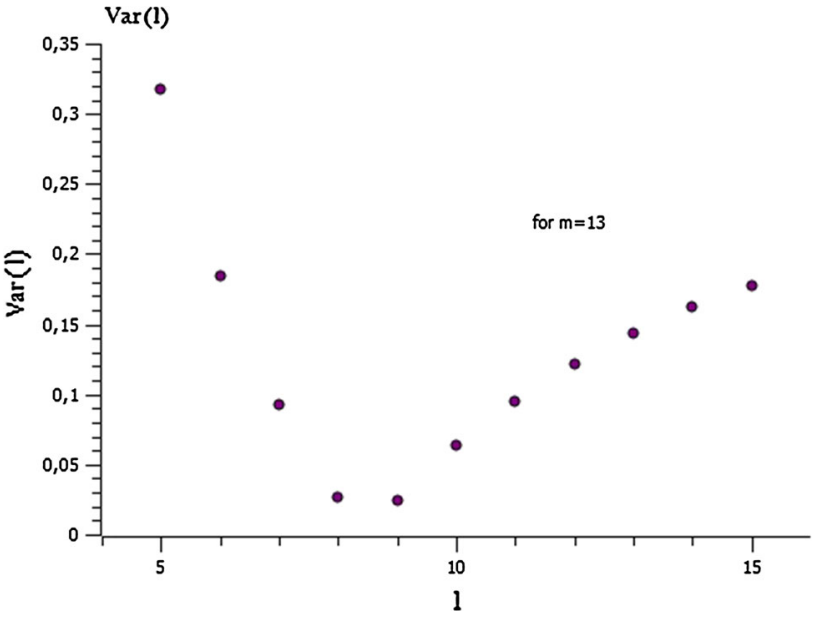

Fig. 7 The variation of the $G$ parameter for $m=13$

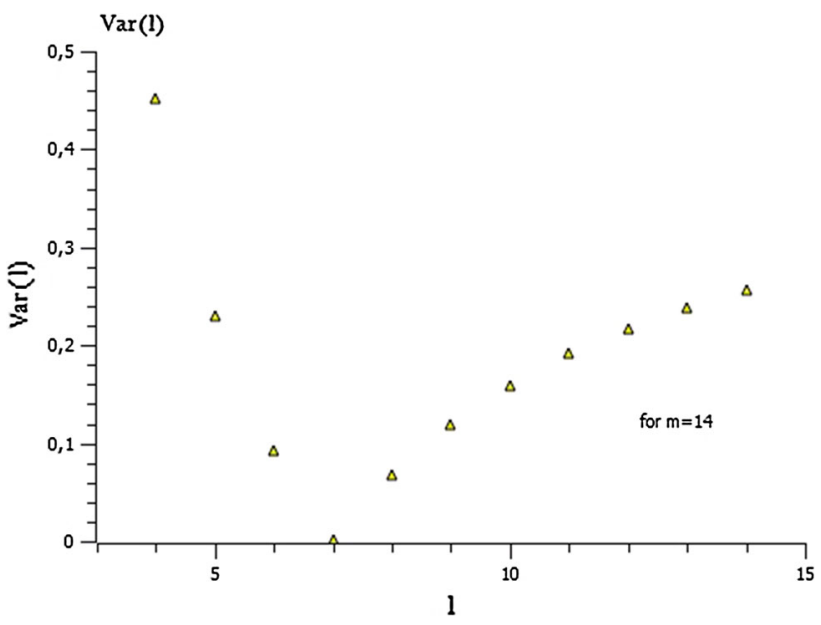

Fig. 8 The variation of the $G$ parameter for $m=14$

The variation of the $G$ parameter exceeds our limits, and the condition of the volume contraction of the "inner space" is not met.

6. For $m=15$ : the variation of the $G$ and the "internal space" parameters are zero for $l=6$, see Fig. 9,

$$
\begin{aligned}
& H=\frac{1}{6}, \\
& h=-\frac{1}{3}, \\
& \text { Int }=0, \\
& \text { Var }=0 .
\end{aligned}
$$

This is the second case with a zero variation of $G$. The exact values of the "Hubble-like" parameters $(H=$ $-\frac{1}{2} h$ ) have simple and compact forms.

Now we will reverse our method and look for the solutions with minimal variation of $G$ for fixed $l$ instead of $m$. The 


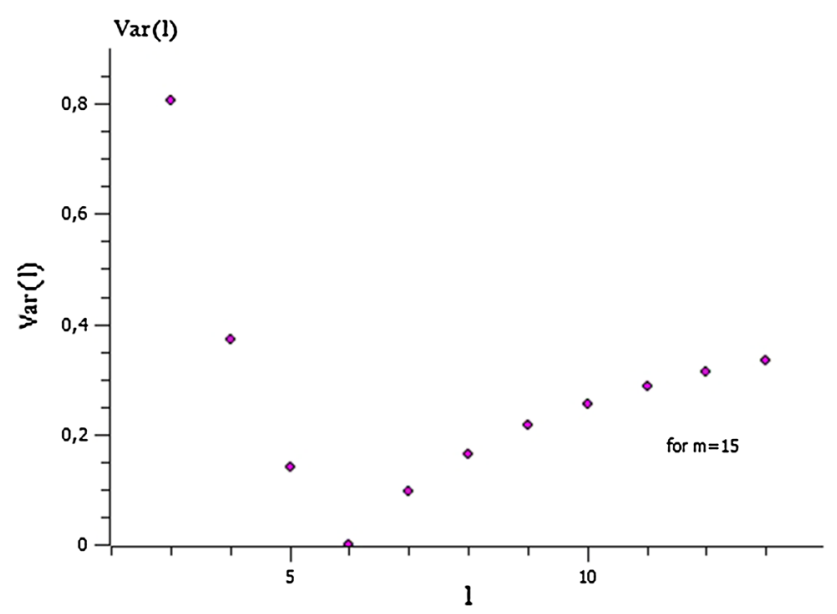

Fig. 9 The variation of the $G$ parameter for $m=15$

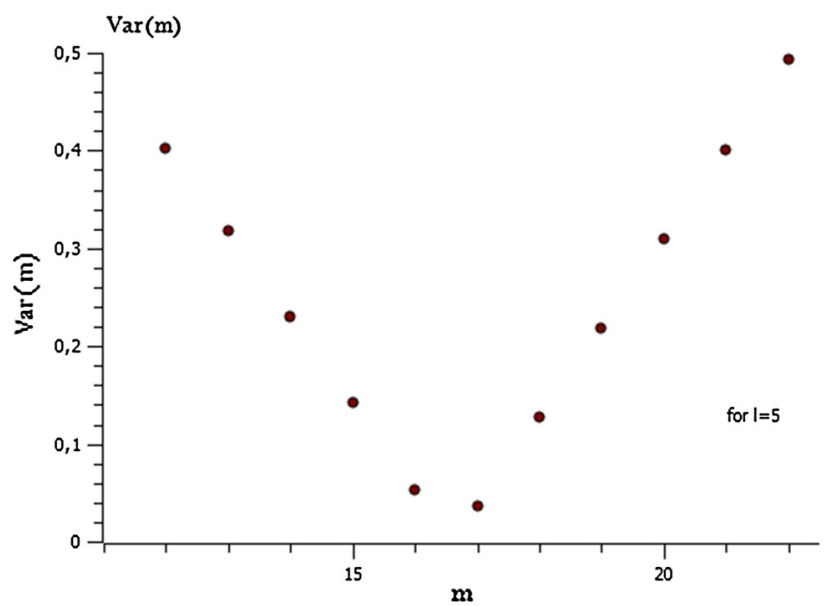

Fig. 10 The variation of the $G$ parameter for $l=5$

calculations lead to the following rule: the lesser is $l$ the greater is an appropriate $m$ which gives the minimum of Var. As we consider $l \geq 3$ and for $l=6$ the solution with minimal variation of the $G$ parameter is already found, and we should examine only three cases.

1. For $l=5$ : the variation of the $G$ parameter is minimal for $m=17$, see Fig. 10,

$H \approx 0.1447364880$,

$h \approx-0.4041874693$,

Int $\approx 0.005373486$,

Var $\approx 0.017555134$.

None of the desirable conditions are satisfied.

2. For $l=4$ : the variation of the $G$ parameter is minimal for $m=20$, see Fig. 11,

$$
\begin{aligned}
H & \approx 0.1220672556, \\
h & \approx-0.5176845111,
\end{aligned}
$$

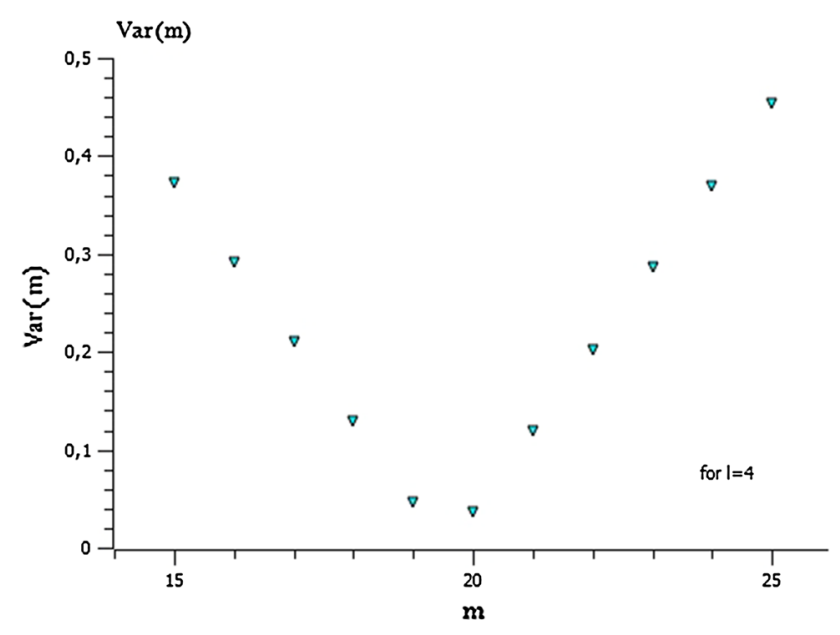

Fig. 11 Variation of $G$ for $l=4$

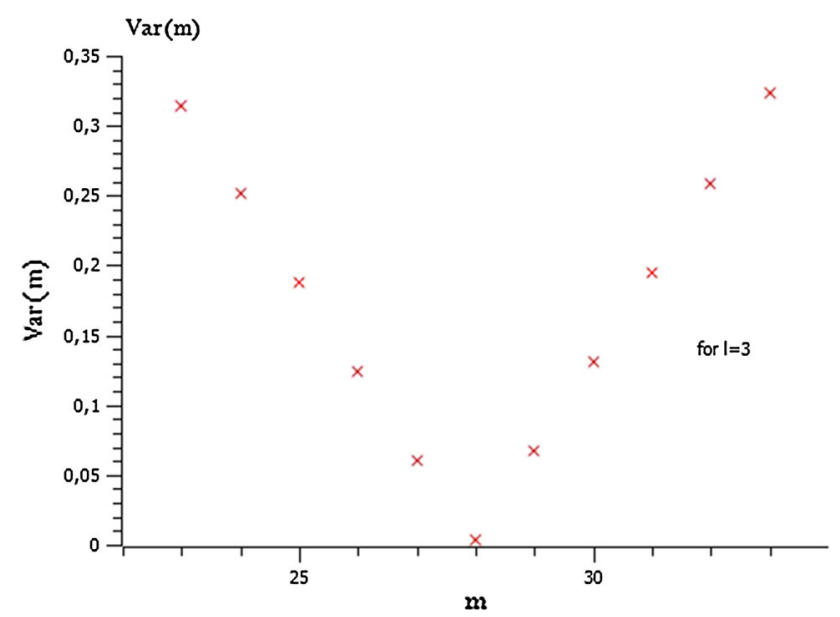

Fig. 12 The variation of the $G$ parameter for $l=3$

$$
\begin{aligned}
& \text { Int } \approx 0.004405301, \\
& \text { Var } \approx 0.03608913 .
\end{aligned}
$$

The amount of variation is too high and the "internal space" parameter is positive.

3. For $l=3$ : the variation of the $G$ parameter is minimal for $m=28$, see Fig. 12,

$$
\begin{aligned}
H & \approx 0.09202826388, \\
h & \approx-0.7765606872, \\
\text { Int } & \approx 0.000272078, \\
\text { Var } & \approx 0.00295646
\end{aligned}
$$

The "internal space" parameter is also positive and the variation of the $G$ parameter exceeds the limits imposed.

Thus, in this subsection we have obtained cosmological solutions for $m>9$, which satisfy all four conditions for the following cases: 
1. $m=11, l=16$ (zero variation of $G)$;

2. $m=12, l=11$;

3. $m=15, l=6$ (zero variation of $G)$.

It should be noted that for $m=3$ and $l=2$ the solution with $H \approx 0.750173$ and $h \approx-0.541715$ was found earlier in [16]. For this solution we have a contracting "internal space" but the variation of $G$ is a huge one $(\dot{G} / G$ is of the order of the Hubble parameter). Recently, an exact analytic form of this solution was obtained in [17].

\section{Conclusions}

We have considered the $(n+1)$-dimensional EGB model. By using the ansatz with diagonal cosmological type metrics, we have found solutions with an exponential dependence of the scale factors with respect to the "synchronous-like" variable $\tau$.

In the cosmological case $(w=-1)$ these solutions describe an exponential expansion of "our" 3-dimensional factor space with the Hubble parameter $H>0$ and obey the observational constraints on the temporal variation of the effective gravitational constant $G$. Any solution describes an $(m-3+l)$-dimensional "internal space", which is anisotropic: it is expanding in $(m-3)$ dimensions with the Hubble rate $H>0$ and contracting in $l$ dimensions.

These solutions were found (in numerical or analytical forms) for the following cases:

1. $m=9, l \geq 2680$ (variation of $G$ tends to 0 as $l \rightarrow+\infty$ );

2. $m=11, l=16$ (variation of $G$ is zero);

3. $m=12, l=11$;

4. $m=15, l=6$ (variation of $G$ is zero).

Thus, we have shown that it is possible in the framework of the EGB model to describe the accelerated expansion of the 3-dimensional factor space with sufficiently small (or even zero) value of the variation of the effective gravitational constant $G$. For the case $w=1$ we have obtained as a byproduct a family of static configurations which may be of interest within some other possible applications.

Here we have considered a gravitational model in more than 4 dimensions. In such a case the Gauss-Bonnet term gives non-trivial contributions to the generalized Einstein field equations. In particular, we have shown that there are cosmological solutions in agreement with observations when "projected" on the $(3+1)$-dimensional physical space-time. For the sake of simplicity, we restrict ourselves to vacuum solutions in multi-dimensional gravity with the GaussBonnet term. Such an ansatz may be considered as a part of a general "geometrical program" aimed at the explanation of dark energy in 4-dimensional space, e.g. by using extra dimensions and modified equations of motion just without matter sources. This is a first step. The inclusion of matter sources (e.g. an anisotropic fluid) will be the next step, as a subject of a subsequent publication.

Open Access This article is distributed under the terms of the Creative Commons Attribution 4.0 International License (http://creativecomm ons.org/licenses/by/4.0/), which permits unrestricted use, distribution, and reproduction in any medium, provided you give appropriate credit to the original author(s) and the source, provide a link to the Creative Commons license, and indicate if changes were made.

Funded by SCOAP ${ }^{3}$.

\section{References}

1. B. Zwiebach, Curvature squared terms and string theories. Phys. Lett. B 156, 315 (1985)

2. D. Gross, E. Witten, Superstrings modifications of Einstein's equations. Nucl. Phys. B 277, 1 (1986)

3. D.J. Gross, J.H. Sloan, The quartic effective action for the heterotic string. Nucl. Phys. B 291, 41 (1987)

4. R.R. Metsaev, A.A. Tseytlin, Two loop beta function for the generalized bosonic sigma model. Phys. Lett. B 191, 354 (1987)

5. R.R. Metsaev, A.A. Tseytlin, Order alpha-prime (two loop) equivalence of the string equations of motion and the sigma model Weyl invariance conditions: dependence on the dilaton and the antisymmetric tensor. Nucl. Phys. B 293, 385 (1987)

6. S. Nojiri, S.D. Odintsov, Introduction to modified gravity and gravitational alternative for Dark Energy. Int. J. Geom. Meth. Mod. Phys. 4, 115-146 (2007). arXiv:hep-th/0601213

7. G. Cognola, E. Elizalde, S. Nojiri, S.D. Odintsov, S. Zerbini, Oneloop effective action for non-local modified Gauss-Bonnet gravity in de Sitter space. arXiv:0905.0543

8. H. Ishihara, Cosmological solutions of the extended Einstein gravity with the Gauss-Bonnet term. Phys. Lett. B 179, 217 (1986)

9. N. Deruelle, On the approach to the cosmological singularity in quadratic theories of gravity: the Kasner regimes. Nucl. Phys. B 327, 253-266 (1989)

10. E. Elizalde, A.N. Makarenko, V.V. Obukhov, K.E. Osetrin, A.E. Filippov, Stationary vs. singular points in an accelerating FRW cosmology derived from six-dimensional Einstein-Gauss-Bonnet gravity. Phys. Lett. B 644, 1-6 (2007). arXiv:hep-th/0611213

11. K. Bamba, Z.-K. Guo, N. Ohta, Accelerating cosmologies in the Einstein-Gauss-Bonnet theory with dilaton. Prog. Theor. Phys. 118, 879-892 (2007). arXiv:0707.4334

12. A. Toporensky, P. Tretyakov, Power-law anisotropic cosmological solution in $5+1$ dimensional Gauss-Bonnet gravity. Gravit. Cosmol. 13, 207-210 (2007). arXiv:0705.1346

13. I.V. Kirnos, A.N. Makarenko, S.A. Pavluchenko, A.V. Toporensky, The nature of singularity in multidimensional anisotropic GaussBonnet cosmology with a perfect fluid. Gen. Relativ. Gravit. 42, 2633-2641 (2010). arXiv:0906.0140

14. S.A. Pavluchenko, A.V. Toporensky, A note on differences between $(4+1)$ - and $(5+1)$-dimensional anisotropic cosmology in the presence of the Gauss-Bonnet term. Mod. Phys. Lett. A 24, 513$521(2009)$

15. I.V. Kirnos, A.N. Makarenko, Accelerating cosmologies in Lovelock gravity with dilaton. Open Astron. J. 3, 37-48 (2010). arXiv:0903.0083

16. D.A. Ratanov, Multidimensional cosmology in the model of gravitation with Gauss-Bonnet adding. Magister dissertation (under the supervision of V.D. Ivashchuk). (Inst. of Gravit. and Cosmol., PFUR, Moscow, 2011), pp. 1-30. (In Russian) 
17. D. Chirkov, S. Pavluchenko, A. Toporensky, Exact exponential solutions in Einstein-Gauss-Bonnet flat anisotropic cosmology. Mod. Phys. Lett. A 29, 1450093 (2014). arXiv:1401.2962

18. A.G. Riess et al., Observational evidence from supernovae for an accelerating universe and a cosmological constant. Astron. J. 116, 1009-1038 (1998)

19. S. Perlmutter et al., Measurements of omega and lambda from 42 high-redshift supernovae. Astrophys. J. 517, 565-586 (1999)

20. M. Kowalski, D. Rubin et al., Improved cosmological constraints from new, old and combined supernova datasets. arXiv:0804.4142

21. K.A. Bronnikov, V.D. Ivashchuk, V.N. Melnikov, Time variation of gravitational constant in multidimensional cosmology. Nuovo Cimento B 102, 209-215 (1998)

22. J.-M. Alimi, V.D. Ivashchuk, S.A. Kononogov, V.N. Melnikov, Multidimensional cosmology with anisotropic fluid: acceleration and variation of $G$. Gravit. Cosmol. 12(2-3), 173-178 (2006)

23. V.D. Ivashchuk, S.A. Kononogov, V.N. Melnikov, M. Novello, Non-singular solutions in multidimensional cosmology with perfect fluid: acceleration and variation of $G$. Gravit. Cosmol. 12(4), 273-278 (2006). arXiv:hep-th/0610167
24. V.N. Melnikov, Models of $G$ time variations in diverse dimensions. Front. Phys. China 4, 75-93 (2009)

25. V.D. Ivashchuk, V.N. Melnikov, On time variations of gravitational and Yang-Mills constants in a cosmological model of superstring origin. Gravit. Cosmol. 20(1), 26-29 (2014). arXiv:1401.5491

26. E.V. Pitjeva, Updated IAA RAS planetary ephemerides-EPM2011 and their use in scientific research. Astron. Vestnik 47(5), 419-435 (2013). arXiv:1308.6416

27. P.A.R. Ade et al., Planck Collaboration, Planck 2013 results. I. Overview of products and scientific results. Astron. Astrophys. 571, A1 (2014). arXiv: 1303.5076

28. S.A. Pavluchenko, On the general features of Bianchi-I cosmological models in Lovelock gravity. Phys. Rev. D 80, 107501 (2009). arXiv:0906.0141

29. V.D. Ivashchuk, On anisotropic Gauss-Bonnet cosmologies in $(n+$ 1) dimensions, governed by an $n$-dimensional Finslerian 4-metric. Gravit. Cosmol. 16(2), 118-125 (2010). arXiv:0909.5462

30. V.D. Ivashchuk, On cosmological-type solutions in multidimensional model with Gauss-Bonnet term. Int. J. Geom. Methods Mod. Phys. 7(5), 797-819 (2010). arXiv:0910.3426 